\title{
Imperfect Is Perfect: Expanding Use of Video for Reference and Instruction
}

AMELIA BIRDSALL LOOBY

Dartmouth College, Hanover, NH

amelia.b.looby@dartmouth.edu

Video, both live and recorded, has become a ubiquitous part of our lives. In some ways, this has been freeing and has expanded our capacity and willingness to use video for instruction and reference.

The business librarians at Dartmouth College primarily serve the MBA students of the Tuck School of Business. Traditional instruction occurs at weekly workshops, dubbed QuickTakes, and one-on-one meetings, typically outside of classroom activity.

Prior to the pandemic, we planned to make a series of highly-polished short database demonstration videos, branding them as QuickerTakes. While a QuickTakes workshop typically lasts around 30 minutes and is a live demonstration of several business resources, the QuickerTakes videos were meant to be much shorter, just a few minutes long. We aimed for the perfect search, just the most crucial commentary, with not an "um" to be heard. We completed two QuickerTakes before COVID-19 sent us home, and our everyday relationship to video changed.

Before COVID-19, the technological bar for recording our in-person workshops was too high. While we were sometimes asked for a recording, if a student was unable to attend, we'd offer them a one-onone overview. Now that we're working from home, our QuickTakes have become Zoom presentations by necessity. Recording within Zoom is easy. Using Dartmouth-licensed video editing software, typically some combination of Adobe Premiere Rush and Techsmith Knowmia, it is simple enough to add title sequences and take out long pauses. Over the past six months, the editing process has gotten fasterboth from experience and a more relaxed attitude.

Informally, we have heard from our Dartmouth peers that students do not mind a less-than-perfect video performance. The small flubs show librarian personality and remind students, who may be feeling isolated, that we are in it together.

The pandemic also allowed us to consider other recorded video content. Quick screen capture videos have replaced some of our drop-in reference. Many of the Library's business databases are less than intuitive to use. Students benefit from seeing them in action before trying on their own. While a Zoom call is a great way to demonstrate, there are times that scheduling a meeting takes longer than the actual instruction. In those instances, we record a casual screen capture. The student can watch a highly personalized video when they are ready.

Will we continue to create this video content in the future? I suspect we will. A video does not need to be flawless to meet the needs of our users. Balancing in-person workshops, live video presentations, and recorded video may be the best of all worlds. 